\title{
Hypermethylation reduces the expression of PNPLA7 in hepatocellular carcinoma
}

\author{
XIAOJIAO ZHANG ${ }^{1-3 *}$, JUN ZHANG $^{1 *}$, RUI WANG $^{1,2}$, SHICHENG GUO $^{4}$, \\ HUILU ZHANG ${ }^{1}$, YANYUN MA ${ }^{4}$, QINGMEI LIU ${ }^{4}$, HAIYAN CHU ${ }^{4}$, XIANGHONG XU $^{4}$, \\ YITONG ZHANG ${ }^{1}$, DONGQIN YANG ${ }^{1}$, JIUCUN WANG ${ }^{4}$ and JIE LIU ${ }^{1,2}$ \\ ${ }^{1}$ Department of Digestive Diseases, Huashan Hospital, Fudan University, Shanghai 200040; \\ ${ }^{2}$ Department of Immunology of Shanghai Medical School, Institutes of Biomedical Sciences, \\ Collaborative Innovation Center of Genetics and Development, Fudan University, Shanghai 200032; \\ ${ }^{3}$ Department of Chemistry and ${ }^{4}$ Ministry of Education (MOE) Key Laboratory of Contemporary Anthropology, \\ State Key Laboratory of Genetic Engineering, School of Life Sciences, Fudan University, Shanghai 200433, P.R. China
}

Received March 30, 2015; Accepted May 5, 2016

DOI: $10.3892 / 01.2016 .4660$

\begin{abstract}
Liver cancer has a high morbidity and mortality rate, and is one of the most common types of cancer in men. PNPLA7 is a member of the patatin-like phospholipase domain-containing protein family which is involved in triglyceride hydrolysis, energy metabolism and lipid droplet metabolism. The liver is the most important energy metabolism organ; whether PNPLA7 is deregulated in liver cancer has not been previously reported. In the present study, reverse transcription-quantitative polymerase chain reaction and subsequent methylation analysis provided evidence that PNPLA7 is down-regulated in hepatocellular carcinoma (HCC) cell lines and tissue samples, via the mechanism of transcriptional silencing by promoter hypermethylation. These results may provide novel insights for HCC diagnosis.
\end{abstract}

\section{Introduction}

Liver cancer has a high morbidity and mortality rate (1), and is one of the most common types of cancer in men.

Correspondence to: Dr Jie Liu, Department of Digestive Diseases, Huashan Hospital, Fudan University, 12 Middle Wulumuqi Road, Shanghai 200040, P.R. China

E-mail: jieliu@fudan.edu.cn

Dr Jiucun Wang, Ministry of Education (MOE) Key Laboratory of Contemporary Anthropology, State Key Laboratory of Genetic Engineering, School of Life Sciences, Fudan University, 2005 Songhu Road, Shanghai 200433, P.R. China

E-mail: theresajcwang@gmail.com

${ }^{*}$ Contributed equally

Key words: hypermethylation, demethylation, PNPLA7, hepatocellular carcinoma
Hepatocellular carcinoma (HCC) is the major histological subtype among primary liver cancer cases, accounting for $70-85 \%$ of the total live cancer burden worldwide $(2,3)$. Liver cancer may be a result of multiple factors and it develops in a multistep process. At present, there are no effective methods for treatment. Although a number of genetic alterations have been reported in the literature, including up- or down-regulated genes (4-6), the overall underlying mechanism remains unknown. It is widely recognized that epigenetic alterations such as methylation and acetylation contribute to carcinogenesis $(7,8)$. Therefore, further exploration of the gene epigenetic changes in HCC are required.

Patatin glycoprotein is highly expressed in mature potato tubers and is a non-specific, lipid acylhydrolase (9). Patatin-like phospholipase domain-containing protein family (PNPLAs) has been identified in a number of species varying from bacteria to human $(10,11)$. PNPLA7, also termed NTE-related 1 (NTE-R1) or NRE, is a member of the PNPLAs family, which is conserved protein in mice, rats and humans (12). It serves key roles in triglyceride hydrolysis, energy metabolism, lipid droplet (LD), and in regulation of adipocyte differentiation $(10,13)$.

There is a marked difference between tumor cells and normal cells in terms of their metabolic patterns, therefore, genes that affect energy metabolism may be potential targets for tumor treatment or diagnosis. It is well recognized that the liver is the most important metabolism organ; whether PNPLA7 is deregulated in HCC has not been previously reported. In the present study, evidence is provided that PNPLA7 was down-regulated in HCC through hypermethylation of its promoter. These findings indicate that PNPLA7 is a potential biomarker for HCC diagnosis.

\section{Materials and methods}

Human tissues and cell lines. HCC tissue samples and the corresponding adjacent non-cancerous tissues were obtained from 52 patients hospitalized in Huashan Hospital 
(Shanghai, China). The study was approved by the Human Research Review Committee of Huashan Hospital and written informed consent was obtained from all patients. Each sample was immersed in RNAlater (Ambion, Ahustin, TX, USA) and stored at $-20^{\circ} \mathrm{C}$ until use. HL-7702 (L02), Huh7, SMMC-7721, HCCLM-6, QGY-7703, HepG2215, and HepG2 cell lines were purchased from the Cell Bank of the Chinese Academy of Sciences (Shanghai, China). The cells were cultured in Dulbecco's modified Eagle's medium with high glucose (DMEM-h) (Gibco; Thermo Fisher Scientific, Inc., Gaithersburg, MD, USA) supplemented with $10 \%$ fetal bovine serum (FBS), in a humid atmosphere with $5 \% \mathrm{CO}_{2}$ at $37^{\circ} \mathrm{C}$.

DNA preparation, RNA extraction, reverse transcription-quantitative polymerase chain reaction $(R T-q P C R)$. Genomic DNA of the cell lines was isolated by QIAamp DNA Blood Mini Kit (Qiagen, Hilden, Germany), according to the manufacturer's instructions. Total RNA was isolated using TRIzol Reagent (Sigma-Aldrich, St. Louis, MO, USA). First-strand cDNA was synthesized from $500 \mathrm{ng}$ total RNA using the high-capacity cDNA reverse transcription kit (Applied Biosystems, Foster City, CA, USA). Quantitative PCR was performed using an Applied Biosystems 7900 Prism real-time PCR system and SYBR Premix Ex Taq (Takara, Dalian, Japan), in accordance with the manufacturer's protocol. Quantitative PCR primers were as follows: PNPLA7, F 5'-GGAAAAGCGTGATGG TTGC-3' and R 5'-GAGCAGGTCCTTCTTGGCA-3'; and GAPDH, F 5'-CAGGGCTGCTTTTAACTCTGGTAA-3' and R 5'-ACTTGATTTTGGAGGGATCTCGCT-3'. Cycling conditions were as follows: $95^{\circ} \mathrm{C}$ for $3 \mathrm{~min}$ followed by 40 cycles of $95^{\circ} \mathrm{C}$ for $15 \mathrm{sec}$ and $60^{\circ} \mathrm{C}$ for $1 \mathrm{~min}$.

Treatment of cells with 5-Aza-dC and trichostatin A (TSA). 5-Aza-dC (Sigma-Aldrich) and TSA (Sigma-Aldrich) were used for demethylation assay. HepG2 cells $\left(5 \times 10^{5}\right.$ cells/well) were seeded in $60 \mathrm{~mm}$ dishes. When the cells reached $30 \%$ coverage, the demethylation agent $5-\mathrm{Aza}-\mathrm{dC}$ was added to the fresh medium at a concentration of $3 \mu \mathrm{M}$. After 4 days, TSA was added at a concentration of $0.5 \mu \mathrm{M}$. The cells were harvested on the 5th day for the extraction of RNA and DNA. The control cells were incubated without 5-aza-dC and TSA.

Bisulfite-sequencing PCR (BSP) analyses. The bisulfite conversion and PCR analyses were performed as described previously (14). BSP primers used for PNPLA7 were F 5'-GTG TAGATTAAGGAGATGGTTT-3' and R 5'-TACTTTTCCAAA TTATCAAAATC-3'. Cycling conditions were as follows: $94^{\circ} \mathrm{C}$ for $3 \mathrm{~min}$; $40 \mathrm{cycles}$ of $94^{\circ} \mathrm{C}$ for $15 \mathrm{sec}, \sim 54^{\circ} \mathrm{C}$ for $20 \mathrm{sec}\left(56^{\circ} \mathrm{C}\right.$ for the 1 st cycle, $54^{\circ} \mathrm{C}$ for the $2 \mathrm{nd}$ cycle and $52^{\circ} \mathrm{C}$ for the remaining cycles) and $72^{\circ} \mathrm{C}$ for $30 \mathrm{sec} ; 72^{\circ} \mathrm{C}$ for $1 \mathrm{~min}$; and $4^{\circ} \mathrm{C}$ thereafter. Then the PCR products were subjected to TA cloning then sent to Invitrogen (Thermo Fisher Scientific, Inc.) for first-generation sequencing (Sanger method). Sequence data was analyzed using Chromas 2.23 (Technelysium Pty Ltd., Brisbane, Australia) and CpGviewer 6.4 (http://dna.leeds.ac.uk/cpgviewer/).

Immunofluorescence. L02 cells (4x105/well) were seeded in a $20 \mathrm{~mm}$ glass bottom cell culture dish and cultured overnight. The cells were washed with PBS and then fixed in $10 \%$ formaldehyde for $30 \mathrm{~min}$ at room temperature. After 3 washes with
PBS, the cells were treated with $0.5 \%$ Triton-X-100 on ice for 5 min, to permeabilize. After a further 3 times of rinsing with PBS, the dish was blocked with $1 \%$ Albumin from bovine serum and incubated with antibodies of rabbit polyclonal PNPLA7 (dilution, 1:200; cat no. ab121302; Abcam) and subsequently with Alexa Fluor 488 Conjugate anti-rabbit IgG (dilution, 1:1,000; cat no. 4412S; Cell Signaling Technology). After 3 washes with PBS, Hoechst staining solution $(1 \mu \mathrm{g} / \mathrm{ml})$ (Life Technologies, Inc., ThermoFisher Scientific, Inc.) was added to completely cover the cells and the cells were incubated for $10 \mathrm{~min}$ at room temperature in the dark. After a further 3 washes, the cells were imaged with a LSM 710 confocal microscope (Carl Zeiss AG, Oberkochen, Germany).

Statistical analysis. Student's t-test was performed to identify statistical significance. Statistical analysis was performed using SPSS software, version 20 (SPSS, Inc., Chicago, IL, USA).P $<0.05$ was considered to indicate a statistically significant difference. GraphPad Prism 5 (GraphPad, San Diego, CA, USA) was used to draw the figures.

\section{Results}

PNPLA7 was down-regulated in HCC tissues and cell lines. Transcriptional expression of PNPLA7 was evaluated in 52 pairs of HCC samples by RT-PCR. After the expression levels were normalized against GAPDH levels, the results showed that PNPLA7 expression was dramatically decreasedin the HCC samples compared to normal controls in the tissue pairs studied ( $\mathrm{P}<0.01$, Fig. 1A). PNPLA7 expression was also significantly down-regulated in all six HCC cell lines compared to the normal liver cell line, L02 ( $\mathrm{P}<0.01$, Fig. 1B).

Hypermethylation of PNPLA7 promoter existed in HCC cell lines. DNA methylation is often correlated with deregulation of variety of genes. The DNA methylation statue of the region (chr9:140446407-140447247) was determined with a BSP-based assay on 6 HCC cell lines and 1 normal liver epithelial cell line. As presented in Fig. 2, the average methylation rate in the $6 \mathrm{HCC}$ cell lines $(85.8 \%)$ was considerably higher than that observed in the normal liver epithelial cell line L02 (3.3\%) $(\mathrm{P}<0.001)$, indicating that the promoter region was hypermethylated in $\mathrm{HCC}$.

PNPLA7 expression was restored after 5-Aza-docy treatment. To confirm the correlation between PNPLA7 expression and the methylation status of its promoter, a demethylation experiment was performed. The HepG2 cell line was treated with DNA methyltransferase inhibitor 5-Aza-dC and the histone deacetylase inhibitor TSA. As shown in Fig. 3A, the result of RT-qPCR assay revealed that the expression of PNPLA7 in HepG2 cell line was significantly up-regulated after treatment with 5-Aza-dC and TSA $(\mathrm{P}<0.01)$. In addition, BSP results (Fig. 3B) confirmed that the majority of the methylated sites were demethylated following treatment, and the corresponding methylation rate decreased by $93 \%$ (to 5.56\%) (Fig. 3C; $\mathrm{P}<0.01)$.

Subcellular location of PNPLA7 in HCC cells. Confocal imaging was performed and indicated that PNPLA7 was 
A

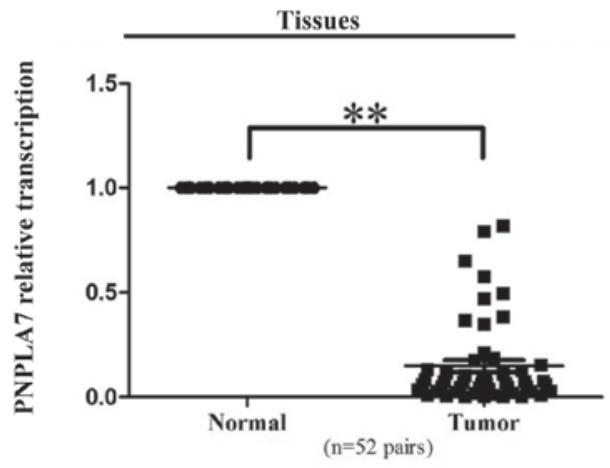

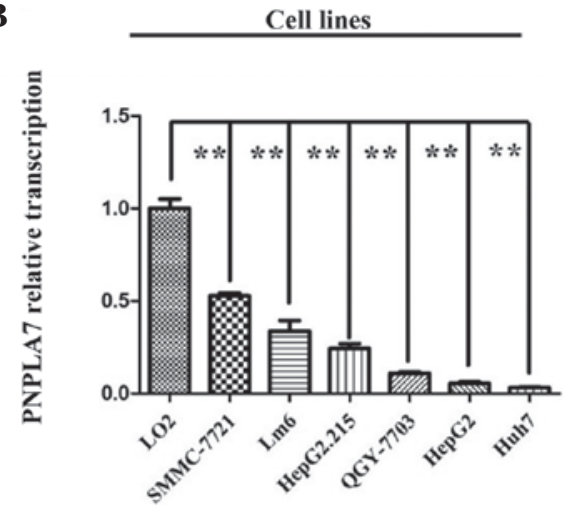

Figure 1. PNPLA7 was down-regulated in HCC tissues and cell lines. (A) RNA expression of PNPLA7 was evaluated in 52 pairs of HCC tissues. The $y$ axis is plotted on a logarithmic scale. (B) mRNA expression levels of PNPLA7 in 6 HCC cancer cell lines and 1 normal cell line. Data represent mean \pm standard deviation from 3 independent experiments. Relative mRNA expression of PNPLA7 in corresponding HCC tissues and the L02 cells was normalized to 1. ${ }^{* *} \mathrm{P}<0.01$. PNPL7, patatin-like phospholipase domain-containing protein 7; HCC, hepatocellular carcinoma.

A

102

SMMC-7721

HepG2.215

QGY-7703

HepG2
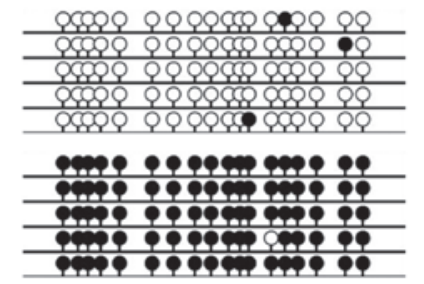

6

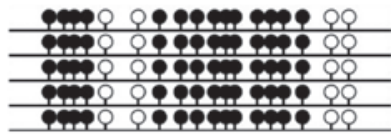

B

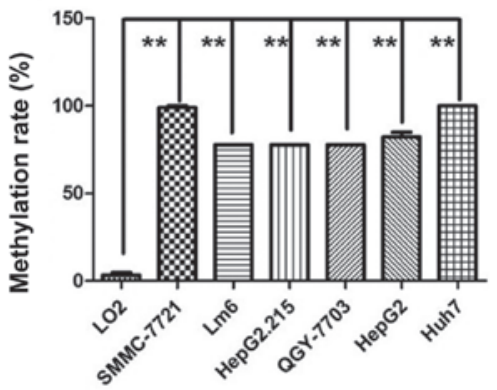

Huh7

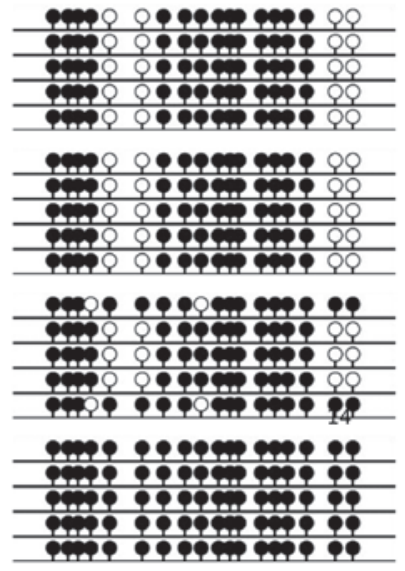

Figure 2. Hypermethylation of PNPLA7 promoter existed in HCC cell lines. (A) Methylation status of the fragment was examined by BSP in HCC cell lines, SMMC-7721, Lm6, HepG2.215, QGY-7703, HepG2, Huh7, and in normal liver cell line LO2. Densitometric analysis of methylation rate of the tested fragment in different cell lines based on BSP results. (B) Methylation rates in LO2, SMMC-7721, Lm6, HepG2.215, QGY-7703, HepG2 and Huh7 were 3.3, 98.9, 77.8, 77.8, 77.8, 82.2 and $100 \%$, respectively. ${ }^{* *} \mathrm{P}<0.01$. PNPL7, patatin-like phospholipase domain-containing protein 7; HCC, hepatocellular carcinoma.

mainly located in plasma membrane and partly distributed in cytoplasm (Fig. 4).

\section{Discussion}

The present study indicated that deregulation of PNPLA7 was associated with HCC and identified that hypermethylation resulted in reduced expression levels of PNPLA7 in HCC.

PNPLAs are considered to be a divergent family, the majority if which have a highly conserved orthologue in several mammalian species (10). PNPLA family members share a protein domain discovered initially in patatin, which is a lipid hydrolase with an unusual folding topology that differs for diverse substrates such as triacylglycerols, phospholipids, and retinol esters (15).

PNPLA7 is $61 \%$ identical to PNPLA6 in amino acid sequence and has the same domain structure, however, it cannot substitute for PNPLA6 during embryonic development $(10,12)$ and it has a different tissue distribution, richest in lysosome, mitochondrion, nucleus and vacuole, and was regulated by insulin and glucose levels $(16,17)$. Both these proteins are predicted to be regulated by cyclic nucleotide as integral membrane proteins and potent 
A

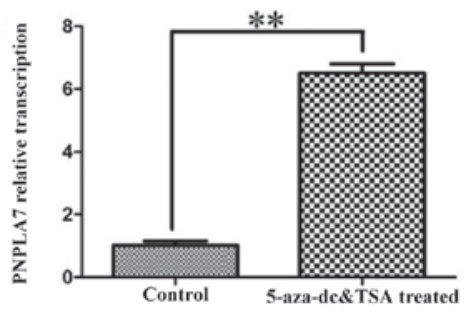

B

C

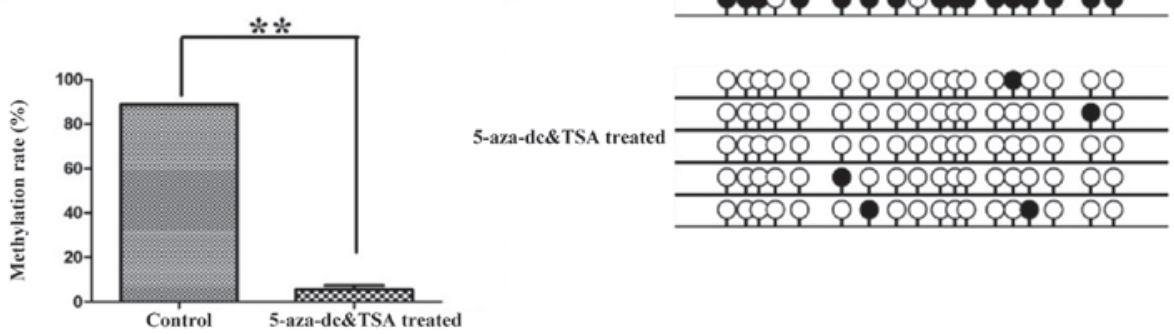

Figure 3. PNPLA7 expression was restored after 5-Aza-docy treatment. (A) PNPLA7 mRNA expression in HepG2 cells with and without demethylation treatment. Data represent mean \pm sd from at least 3 independent experiments, ${ }^{* * *} \mathrm{P}<0.01$. (B) Methylation status of the fragment was assessed using BSP in HepG2 cells with and without demethylation treatment. (C) Densitometric analysis of methylation rate of the tested fragment in HepG2 cells with and without demethylation treatment based on BSP results, " $\mathrm{P}<0.01$. PNPL7, patatin-like phospholipase domain-containing protein 7; HCC, hepatocellular carcinoma; BSP, bisulfite sequencing PCR.

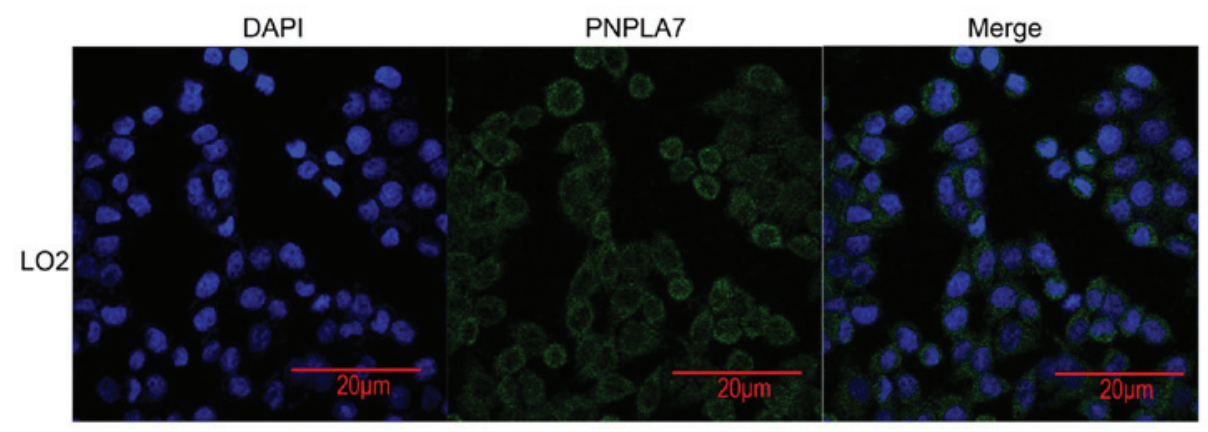

Figure 4. PNPLA7 was mainly located in plasma membrane and partly distributed in cytoplasm. Bar= $20 \mu \mathrm{m}$, and observed with 40x objective lens. PNPL7, patatin-like phospholipase domain-containing protein 7.

lysophospholipase activity, while they showed different sensitivity to organophosphate inhibitors (18).

Other members of PNPLA family were reported to be involved in lipid metabolism and chronic hepatitis $\mathrm{C}$ infection. PNPLA3, particularly, was strongly associated with liver injury and non-alcoholic fatty liver disease (NAFLD) (19-21). However, there are few reports relating to PNPLA7 in human diseases. Vrieze et al (22) mapped $\sim 85,000$ rare nonsynonymous exonic single nucleotide polymorphisms (SNPs) to 17 psychophysiological endophenotypes in 4,905 individuals and identified that PNPLA7 is associated with the endophenotype pleasant difference startle, the difference in startle magnitude between pleasant and neutral images. Therefore, the present study aimed to explore whether PNPLA7 was also associated with liver disease like PNPLA3. Here we observed that PNPLA7 was down-regulated in HCC cell-lines and also in tissue samples.

Promoter hypermethylation induced transcriptional silencing has emerged recently as one important mechanism involved in oncogenesis and cancer development $(23,24)$. In order to understand the molecular mechanism of the down-regulation of PNPLA7 in HCC, the methylation status of $\mathrm{CpG}$ island of PNPLA7 promoter was analyzed and hypermethylation was confirmed in all HCC cell lines. Then
5-Aza-dC, a demethylating agent, was used and this treatment restored PNPLA7 expression in liver cancer cell lines. The results revealed that promoter hypermethylation could result in inhibition of PNPLA7 transcription in HCC.

In conclusion, the present study demonstrated that PNPLA7 levels were dramatically down-regulated in both HCC cell lines and tissues. It was also determined that DNA hypermethylation was the mechanism of the down-regulation of PNPLA7 in HCC. These results may offer a novel insight to identifying cancer markers and understanding the mechanism of hepatocarcinogenesis.

\section{Acknowledgements}

The present study was supported by grants from the Ministry of Science and Technology of China (grant no. 2013CB945401) and the National Natural Science Foundation of China (grant nos. 81125001 and 91129702).

\section{References}

1. Torre LA, Bray F, Siegel RL, Ferlay J, Lortet-Tieulent J and Jemal A: Global cancer statistics, 2012. CA Cancer J Clin 65: $87-108,2015$ 
2. Leong TY and Leong AS: Epidemiology and carcinogenesis of hepatocellular carcinoma. HPB (Oxford) 7: 5-15, 2005.

3. El-Serag HB and Rudolph KL: Hepatocellular carcinoma: Epidemiology and molecular carcinogenesis. Gastroenterology 132: 2557-2576, 2007.

4. Buendia MA and Neuveut C: Hepatocellular carcinoma. Cold Spring Harb Perspect Med 5: a021444, 2015.

5. Lemmer ER, Friedman SL and Llovet JM: Molecular diagnosis of chronic liver disease and hepatocellular carcinoma: The potential of gene expression profiling. Semin Liver Dis 26: 373-384, 2006.

6. Matter MS, Decaens T, Andersen JB and Thorgeirsson SS Targeting the mTOR pathway in hepatocellular carcinoma: Current state and future trends. J Hepatol 60: 855-865, 2014

7. Easwaran H, Tsai HC and Baylin SB: Cancer epigenetics: Tumor heterogeneity, plasticity of stem-like states and drug resistance. Mol Cell 54: 716-727, 2014.

8. Ma L, Chua MS, Andrisani O and So S: Epigenetics in hepatocellular carcinoma: An update and future therapy perspectives. World J Gastroenterol 20: 333-345, 2014.

9. Rydel TJ, Williams JM, Krieger E, Moshiri F, Stallings WC, Brown SM, Pershing JC, Purcell JP and Alibhai MF: The crystal structure, mutagenesis, and activity studies reveal that patatin is a lipid acyl hydrolase with a Ser-Asp catalytic dyad. Biochemistry 42: 6696-6708, 2003.

10. Wilson PA, Gardner SD, Lambie NM, Commans SA and Crowther DJ: Characterization of the human patatin-like phospholipase family. J Lipid Res 47: 1940-1949, 2006.

11. Finn RD, Bateman A, Clements J, Coggill P, Eberhardt RY, Eddy SR, Heger A, Hetherington K, Holm L, Mistry J, et al: Pfam: The protein families database. Nucleic Acids Res 42 (Database Issue): D222-D230, 2014.

12. Winrow CJ, Hemming ML, Allen DM, Quistad GB, Casida JE and Barlow C: Loss of neuropathy target esterase in mice links organophosphate exposure to hyperactivity. Nat Genet 33 477-485, 2003.

13. Kienesberger PC, Lass A, Preiss-Landl K, Wolinski H, Kohlwein SD, Zimmermann R and Zechner R: Identification of an insulin-regulated lysophospholipase with homology to neuropathy target esterase. J Biol Chem 283: 5908-5917, 2008
14. Herman JG and Baylin SB: Methylation-specific PCR. Curr Protoc Hum Genet Chapter 10: Unit 10.6, 2001.

15. Kienesberger PC, Oberer M, Lass A and Zechner R: Mammalian patatin domain containing proteins: A family with diverse lipolytic activities involved in multiple biological functions. J Lipid Res 50 (Suppl): S63-S68, 2009.

16. Chang PA, Long DX, Sun Q, Wang Q, Bu YQ and Wu YJ: Identification and characterization of a splice variant of the catalytic domain of mouse NTE-related esterase. Gene 417: 43-50, 2008.

17. Richardson RJ, Hein ND, Wijeyesakere SJ, Fink JK and Makhaeva GF: Neuropathy target esterase (NTE): Overview and future. Chem Biol Interact 203: 238-244, 2013

18. Chang PA, Chen YY, Long DX, Qin WZ and Mou XL: Degradation of mouse NTE-related esterase by macroautophagy and the proteasome. Mol Biol Rep 39: 7125-7131, 2012.

19. Kotronen A, Johansson LE, Johansson LM, Roos C, Westerbacka J, Hamsten A, Bergholm R, Arkkila P, Arola J, Kiviluoto T, et al: A common variant in PNPLA3, which encodes adiponutrin, is associated with liver fat content in humans. Diabetologia 52: 1056-1060, 2009.

20. Sookoian S, Castaño GO, Burgueño AL, Gianotti TF, Rosselli MS and Pirola CJ: A nonsynonymous gene variant in the adiponutrin gene is associated with nonalcoholic fatty liver disease severity. J Lipid Res 50: 2111-2116, 2009.

21. Valenti L, Al-Serri A, Daly AK, Galmozzi E, Rametta R, Dongiovanni P, Nobili V, Mozzi E, Roviaro G, Vanni E, et al: Homozygosity for the patatin-like phospholipase-3/adiponutrin I148M polymorphism influences liver fibrosis in patients with nonalcoholic fatty liver disease. Hepatology 51: 1209-1217, 2010

22. Vrieze SI, Malone SM, Pankratz N, Vaidyanathan U, Miller MB, Kang HM, McGue M, Abecasis G and Iacono WG: Genetic associations of nonsynonymous exonic variants with psychophysiological endophenotypes. Psychophysiology 51: 1300-1308, 2014.

23. Jones PA and Laird PW: Cancer epigenetics comes of age. Nat Genet 21: 163-167, 1999.

24. Lopez-Serra P and Esteller M: DNA methylation-associated silencing of tumor-suppressor microRNAs in cancer. Oncogene 31: 1609-1622, 2012. 